\title{
THE RELATION OF THE TASTE OF ACIDS TO THEIR DEGREE OF DISSOCIATION, II
}

\section{BY THEODORE WILLIAM RICHARDS}

Some years ago ${ }^{x}$ it was shown that weak acids have a more strongly acid taste than is demanded by their degree of dissociation. In a recent interesting article upon the taste of acid salts, Kahlenberg ${ }^{2}$ shows that these bodies also have a far stronger taste than one would expect.

Two possible explanations of these phenomena were given in the first paper $:^{3}$ either the act of tasting removes hydrogen ions and thus induces the formation of more, or else the undissociated acid possesses a taste similar to that of hydrogen ions. Kahlenberg ascribes the acid taste in his case to the anion (for instance $\mathrm{HC}_{2} \mathrm{O}_{4}^{\prime}$ ), an interpretation which is impossible in the case of a monobasic acid, because the neutral salts in question are practically tasteless.

While it is undoubtedly true that many undissociated substances (for example sugar) have a strong taste, and that some anions also have a perceptible taste, ${ }^{4}$ it seems to me probable that in the cases of the weak acids in question, the hydrogen ion is the

${ }^{1}$ Richards. Am. Chem. Jour. 20, I2 I (1898). Kastle. Ibid. 466. Kah1enberg. Jour. Phys. Chem. 3, 66 (I899); Zeit. phys. Chem. 29, 343 (I899). E. H. S. Bailey published in I 887 a brief note concerning the taste of acids, but since this was before the acceptance of the dissociation hypothesis, he was puzzled rather by the fact that acetic acid had not so strong a taste as hydrochloric acid than by the present question. (Proc. Kansas Acad. Sci. II, IO.) This paper was known to none of the above authors at the time of writing, and my attention has been kindly called to it by Professor Bailey.

a Kahlenberg. Jour. Phys. Chem, 4, 33 (I900).

${ }^{3}$ Richards. Am. Chem. Jour. 20, I23 (I898).

${ }^{4}$ Höber und Kiesow. Zeit, phys. Chem. 27, 6or (1898). Kahlenberg. loc. cit. 
chief agent, and hence that the first explanation is the true one.

The natural timidity felt by a chemist as regards even a limited excursion into the physiologist's domain, prevented me from offering any more definite hypothesis at the time, but the present occasion seems to warrant a further explanation. The fundamental cause of the sensation of taste is not yet clear; perhaps the most probable view is that the sensation is due to temporary chemical change in the tongue surface. It is clear that osmotic action alone cannot be the cause, for in that case all isotonic solutions should taste alike. The cell walls, it is true, may be permeable to some bodies and impermeable to others; but a cursory glance at the list of strong tasting substances seems to show that if such a partial permeability exists, it must depend upon physico-chemical and not upon purely physical characteristics of the dissolved substances.

Since the object of this paper is merely to show that the irregular phenomena manifested by the taste of acids may be explained in a physico-chemical fashion, the other possible hypotheses need not be considered here. We shall assume, then, that the phenomena of taste are due primarily to chemical reactions taking place in the tongue-surface, and seek to discover if the assumption leads us to inconsistencies as far as the taste of acids is concerned. It is quite possible that taste, like sight, is caused by only a few independent reactions; - that all the variety of tastes may be due simply to the superposition of these sensations, just as all the variety of colors is known to be due simply to violet, green and red. ${ }^{\mathrm{T}}$ The manufacture of scents from a combination of substances whose individual odors everyone condemns, is an analogous case illustrating this possibility. The existence of indol in the oil of jassamine is another similar case in point. It is well known, at least, that the number of true taste sensations is far fewer than is popularly supposed, for many of our so-called tastes are really smells. ${ }^{2}$

\footnotetext{
"See for example "An American Text Book of Physiology." Howell, p. 85 I (I 896$)$.

${ }^{2}$ Ramsay has published an interesting paper on the relation of smell to
} 
However all this may be, it is certain that the sourness of acids is one of the simplest and most powerful of tastes, and hence one best adapted to furnish material for the consideration of the whole question. If we accept the theory of electrolytic dissociation, we cannot doubt that the taste of strong acids, much diluted with water, is due to the hydrogen ion; although the tastes of many other ions are so feeble that conclusions as regards origin of the peculiar tastes of salts are rather hazardous.

How then does the hydrogen ion effect the supposed chemical change causing the sour taste? There are of course two ways in which the acid may act:-either catalytically, or else by direct combination. If the action is catalytic, the change in the concentration of the hydrogen ion in the neighborhood of the nerve terminal during the reaction cannot be large, for any acid existing in the tongue-surface must be exceedingly weak. Hence taste should be a fairly accurate indicator of the concentration of the hydrogen ion. This assumption then leads us to a result in opposition to the facts; and in order to maintain it one must make the unlikely extra assumption that a complex body is capable of producing the simple effect caused by ionized hydrogen.

On the other hand, if, as seems more probable, the tastereaction is one into which the hydrogen ion actually enters, such as the action of a strong acid on the salt of a weak one, or the neutralization of a nitrogenous organic base, ${ }^{x}$ it is clear that the act of tasting must remove ionized hydrogen from the liquid tasted. The removal of this ion must of course upset the equilibrium, and immediately more of the acid which is being

the chemical nature of the odoriferous body. A similar study of taste would be interesting also; - for example, a comparative study of the sweetness of polyatomic alcohols, or of the relative effect of the ketone and the aldehyde group in the sugars, etc. Professor Ramsay's paper was printed in the Proceedings of the Glasgow Phil. Soc, a number of years ago, and was partly reprinted in Nature, 26, I87 (I882).

'An interesting paper by Bugarszky and Lieberman [Pflüger's Archiv, 72, 5 I ( 1898$)]$ indicates a class of reactions which may be the important ones in this case. I am greatly indebted to Mr. L. J. Henderson, of the Harvard Medical School, for his kindness in preparing for me an abstract of this article. 
tasted must be dissociated, according to the law of mass action. The newly ionized hydrogen must also be removed, allowing more to form, and so on. Hence a weak acid may cause a far greater change on the tongue-surface than corresponds to the concentration of the hydrogen ion in the pure acid; and this conclusion is wholly in accord with the facts.

Obviously, if acetic acid should yield up all its hydrogen ions to the tongue, it would taste as strong as an equivalent solution of hydrochloric acid. In such a case, one might as well attempt to determine the degree of dissociation by titration with baric hydroxide as by taste. The fact that weak acetic acid really tastes less sour than hydrochloric acid would tend to show that a state of equilibrium is reached before all the acetic acid has been dissociated; or else that the cell walls limit the speed of the reaction. The data are too inaccurate to make a quantitative calculation of value, but the principle involved is sufficiently evident. It is worthy of note that at least the order of sourness of weak acids is qualitatively correct, a fact which accords entirely with the present explanation.

Of course, precisely the same train of thought applies to weak acid salts; and indeed by analogy the explanation may be extended to the taste of hydroxyl ions. In this case quantitative measurement is even less certain because of the necessary presence of carbon dioxide. The explanation is in accord with the fact that hydrogen and hydroxyl ions possess a far stronger taste than most other.ions; for it is obvious that these two should be more capable than any other ions of causing reactions in bodies likely to be present in the tongue-surface.

Physiologists have decided, I believe, that the different kinds of taste are perceived by different regions of the tongue. Of course the respective regions must be so constituted that the appropriate chemical reaction takes place near the nerve center fitted to receive the impulse. This fact may be used as an argument in favor of the present point of view; for unless there is some other disturbing factor, acid salts are said to be indistinguishable frotn pure acids by taste. 
Of course other reactions, such as the formation of glucosides, must explain other tastes; but it is obvious that in these cases no such cumulative effect could be observed.

On the other hand, strong solutions of all kinds may easily exhaust from the tongue-surface itself the bodies capable of yielding the necessary reactions; hence the chemical explanation of taste elucidates also the partial paralysis of taste produced by concentrated solutions.

Thus none of the observed phenomena concerning the sour taste seem to be opposed to the hypothesis that weak acids and acid salts give a stronger taste than that which corresponds to their degree of ionization simply because hydrogen is deionized in the act of tasting. The other possible hypotheses of taste have not been disproved, because neither proof nor disproof is possible in the present state of our knowledge of physiological chemistry; but at least it has been shown that a satisfactory chemical theory of this particular taste is possible.

Harvard University, Cambridge, Mass., Feb. I4, 1900. 